Voix et Images

volxetimages

\title{
La Question de l’énonciation en poésie : Saint-Denys Garneau
}

\section{Jean Fisette}

Volume 2, numéro 3, avril 1977

Jean Éthier-Blais

URI : https://id.erudit.org/iderudit/200071ar

DOI : https://doi.org/10.7202/200071ar

Aller au sommaire du numéro

Éditeur(s)

Les Presses de l'Université du Québec

ISSN

0318-9201 (imprimé)

1705-933X (numérique)

Découvrir la revue

Citer cet article

Fisette, J. (1977). La Question de l'énonciation en poésie : Saint-Denys Garneau.

Voix et Images, 2(3), 375-389. https://doi.org/10.7202/200071ar d'utilisation que vous pouvez consulter en ligne.

https://apropos.erudit.org/fr/usagers/politique-dutilisation/ 


\section{La Question de l'énonciation en poésie : Saint-Denys Garneau}

Nous avons choisi d'aborder la question de l'énonciation; plus que d'un simple sujet de réflexion, il s'agit là d'un des grands problèmes qui se posent actuellement à la sémiotique. Le champ de recherche est d'ailleurs tellement vaste, et les solutions proposées, tellement floues et fragmentaires, qu'on se limitera, pour l'essentiel de cet exposé, à analyser un texte, à y déchiffrer, à partir d'un minimum d'instruments méthodologiques, les modes d'inscription du sujet. Ce qui nous permettra d'inférer quelques propositions générales concernant le domaine de la poétique.

Que recouvre ce terme d'énonciation? II ne faudrait surtout pas y voir une simple variante, sous des parements renouvelés, de la vieille interrogation portant sur les relations de cause à effet à établir entre la vie personnelle, l'existence biographique d'un écrivain et son œuvre. Le champ abordé est en un sens, plus restreint, plus problématique certes, mais plus susceptible d'apporter une réponse satisfaisante. Qu'en est-il exactement? On pourrait poser ainsi la question: le poète écrivant, que cherchet-il ? A quel besoin répond cette pratique d'écriture?

Ignorant volontairement, et dès le début, la perspective de la communication avec un quelconque public lecteur (la plupart du temps très difficile à délimiter), nous retrouvons le poète seul, avec les mots et avec lui-même, face à la feuille blanche. Ou plutôt, proposons de voir dans l'écrivain le premier lecteur de son propre texte ${ }^{1}$. Sitôt les rapports entre les divers JE deviennent très complexes.

Nous retenons comme lieu exemplaire la poésie car c'est là que s'inscrit avec le plus d'aisance et comme allant de soi, le JE. Les JE inscrits d'une part et d'autre part, les JE énonciateur et lecteur, voilà non pas une simple alternative, mais un espace délimité, à l'intérieur duquel, nous essaierons de jeter quelque lumière.

Notre hypothèse consiste à affirmer que les figurations successives du JE inscrit supposent un rapport dialectique avec les JE hors texte; ces rapports, croyons-nous, fonctionnent suivant les lois du modèle du narcissisme de sorte que la pratique d'écriture apparaît comme une activité où 
le JE énonciateur se met en scène et, du même coup, se masque sous une représentation de lui-même. Ce jeu, nous le nommons voilement/ dévoilement.

Le texte lui-même, saisi comme un espace graphique, réalise une scène fantasmatique où s'opère le travail du signifiant que l'on pourra analyser par étapes successives, en relevant d'abord une syntagmatique de l'imaginaire - par le biais d'une lecture rhétorique - et, par la suite, en mettant en rapport ces champs imaginaires avec la problématique du sujet.

Si l'on respecte la syntagmatique du texte, on trouvera, comme résultat, deux mouvements de transformation - l'un de l'imaginaire, l'autre du sujet - qui sont contraires, d'où une tension qui assure la cohérence du texte et sa clôture. Le sujet apparaîtra dans son mouvement différent de celui, plus simple, de l'autobiographie, par exemple: au lieu de trouver un MOI qui réalise et objective un JE, ce sera le JE qui s'épuise, se détruit, par des phénomènes de substitution, puis se perd sous l'image implicite du IL, figure par excellence du pouvoir.

Le poème "Autrefois ${ }^{2}$ " renvoie à un essai: "Monologue fantaisiste sur le $\operatorname{mot}^{3}$ " que Saint-Denys Garneau faisait paraître dans la Relève en 1937, l'année même de la parution de Regards et jeux dans l'espace. Je dis “renvoie» car en fait, les deux textes semblent correspondre l'un à l'autre de façon tellement contraignante que le poème apparaît, à la première lecture, comme la réalisation - un montage, dirait-on - des propos tenus dans l'essai, et à l'inverse, l'essai se donne comme une approche herméneutique du poème.

Dans l'essai, on trouve une sorte de figuration mystérieuse qui met en corrélation le couple mot-poème. Remarquons au passage que SaintDenys Garneau y affiche, par le fait même, une position liée à la matérialité du texte, étrangère à un pseudo-mysticisme dont on l'a toujours affublé: ici, c'est vraiment le fabricant de poème qui parle. Je retiens du texte, pour mes besoins, une réduction qui, pour schématique qu'elle soit, me semble rester assez fidèle au texte précité : deux affiliations - à la fois phoniques et sémantiques - sont mises en corrélation: MOI-MORT-MOT et la série: PAROLE-POĖME. L'hypothèse du paragrammatisme qui affleure ici ne surprendra pas; au contraire, elle paraît tout à fait judicieuse chez le poète qui, dans la manipulation des mots, les perçoit autant dans leur matérialité sonore - et la valeur du signifiant - que dans leur contenu socialisé; on pourrait même voir dans ces séries, les fondements préconscients d'une pensée ou d'une imagination; c'est là une question que je laisse ouverte... 
Je cite le dernier paragraphe de l'essai:

Et c'est le mystère du poème. Le mot qui enveloppait tout se voit alors haussé a être enveloppé par tout le poème, c'est à dire un réseau de fils invisibles, de rayons dont le poète est le lieu.

Ce qui nous permet de poser en équivalence à nos deux séries le schème bipolaire: CENTRE-PÉRIMĖTRE ou, en d'autres termes: ENVELOPPÉENVELOPPANT. Or tout le poème "autrefois " met en place un mécanisme pour arriver à l'équivalence suivante: Poème-enveloppant et Moi-enveloppé.

D'autre part, ce paragraphe final que nous avons cité propose, sous le terme de "mystère» un phénomène de transformation qui serait le travail du texte. Ce sont exactement ces opérations que nous cherchons à retracer; à l'analyse, elles se révèlent plus complexes que ne le laisse entendre l'essai.

1 - 1 Autrefois j'ai fait des poèmes

2 Qui contenaient tout le rayon

3 Du centre à la périphérie et au-delà

4 Comme s'il n'y avait pas de périphérie

5 Et comme si j'étais le soleil : à l'entour

mais le centre seul

l'espace illimité

II - 6 C'est qu'on prend de l'élan

7 C'est qu'on acquiert une prodigieuse vitesse de bolide

8 Quelle attraction centrale peut alors

9 Quel dôme de firmament concave qu'on le perce

empêcher qu'on s'échappe

10 Quand on a cet élan pour éclater dans l'Au-delà.

III - 11 Mais on apprend que la terre n'est pas plate

12 Mais une sphère et que le centre n'est pas au milieu

13 Mais au centre

14 Et l'on apprend la longueur du rayon ce chemin

15 Et l'on connaît bientôt la surface

trop parcouru

16 Du globe tout mesuré inspecté arpenté vieux sentier

17 tout battu

IV - 18 Alors la pauvre tâche

19 De pousser le périmètre à sa limite

20 Dans l'espoir à la surface du globe d'une fissure,

21 Dans l'espoir et d'un éclatement des bornes

22 Par quoi retrouver libre l'air et la lumière.

$v-23$ Hélas tantôt désespoir

24 L'élan de l'entier rayon devenu

25 Ce point port sur la surface.

$\mathrm{VI}-26$ Tel un homme

27 Sur le chemin trop court par la crainte du port

28 Raccourcit l'enjambée et s'attarde à venir

29 II me faut devenir subtil

30 Afin de, divisant à l'infime distance

31 De la corde à l'arc, 
32 Créer par ingéniosité un espace analogue à l'Au-delà

33 Et trouver dans ce réduit matière

34 Pour vivre et l'art.

La première moitié du poème est constituée d'une succession de syntagmes qui remplissent la double fonction de fonder le champ de l'imaginaire de la géométrie et de l'épuiser. Précisons ici que nous évitons la formule de "métaphore géométrique" puisque un terme ou une série de termes ne peuvent être métaphoriques que par rapport à un point fixe dont ils s'éloigneraient. Notre lecture ici est immanente au poème et, si l'on proposait de situer le poème dans un rapport distancié à l'essai, le terme de métaphore ne serait pas plus juste puisque le champ imaginaire de la géométrie y figure déjà. On prendra donc ce champ imaginaire de la géométrie comme point de départ de l'analyse, tant sur le plan de l'imaginaire que sur celui de la rhétorique.

La cascade des syntagmes fondant, dans la première moitié du poème le champ de la géométrie apparaît comme un déroulement métonymique, étant enteridu que l'on donne à ce terme la définition proposée par le groupe MU dans sa Rhétorique générale ${ }^{4}$, à savoir la co-inclusion de plusieurs termes dans un même ensemble. Dans le cas que nous analysons, le recours à la figure de la métonymie est d'autant plus juste que la définition classique - "la partie pour le tout " - correspond exactement à la figuration géométrique. Or cette succession de «figures géométriques" sur le plan du signifiant autant que sur celui du contenu, conduit à un épuisement momentané:

Ce point mort sur la surface (v. 25)

Tout comme si la conséquence paradoxale de la nomination en cascade était l'aboutissement, suite à un épuisement, à un point fixe, au vide.

Dans une perspective méthodologique, nous proposons de nommer ce phénomène: concentration. Le premier avantage est d'éviter le terme de "métonymie» qui, après ses multiples emplois depuis une dizaine d'années, est devenu, sinon ambigu, du moins trop polysémique pour demeurer efficace. Le deuxième avantage du terme concentration réside dans sa valeur significative tant sur le plan du contenu que de l'expression.

Reprenons le parcours du texte: apparaît un personnage, le «Tel un homme", figure thématique dont le programme sera de réaliser une expansion à la fois spatiale et temporelle:

Raccourcit l'enjambée et s'attarde à venir (v. 28)

peut-on lire. Le "Tel» de "Tel un homme" annonce un effet métaphorique, réalisant par là une des charnières du texte. Dans la même logique terminologique, nous réservons à la notion de métaphore, le terme d'expansion. Ajoutons que ce terme répond à la définition de la métaphore par le groupe $\mathrm{MU}^{5}$ comme relation entre deux termes appartenant à des 
ensembles différents et, du fait même, comme passage d'un ensemble à l'autre.

Le «Tel " réalise effectivement le passage du champ imaginaire de la géométrie à celui de la marche. Ce champ, fort peu exploité dans le poème - trois vers - institue un paraître, une fiction qui, par rapport au champ de la géométrie, n'est que profusion vers l'extériorité, une percée réalisant la quête d'un modèle logique autre.

Car aussitôt le poème revient au champ imaginaire de la géométrie pour réaliser ce qui auparavant s'était avéré impossible: une duplication de l'espace que l'on peut lire dans les vers:

Créer par ingéniosité un espace analogue à l'Au-delà (v. 32)

La troisième et dernière partie du poème paraît comme une synthèse des deux mouvements que nous avons pu lire précédemment: concentration jusqu'à un réduit et expansion par l'analogie des espaces qui devient possible par la transformation du paraître en être.

Le fonctionnement du poème apparaît donc comme un faire linguistique, c'est-à-dire la production de ce réduit final, à partir des deux types dè mouvement qui précédemment assuraient, l'un après l'autre, la gouverne du texte.

Ajoutons que logiquement tout texte, pour se constituer, doit combiner ces deux types de fonctionnement; comme l'équilibre est rarement atteint - et peu souhaitable en fait - il est théoriquement possible de trouver des textes différents qui réalisent tous les dosages possibles. Dans les extrêmes; on trouvera le texte qui n'arrive pas à se fixer un point d'ancrage (le baroque) d'autre part, le texte qui à force de se comprimer, se déprime jusqu'à l'aphasie: peut-être le classicisme le plus pur.

La particularité du poème de Saint-Denys Garneau que notre grille met en évidence, c'est son fonctionnement sur deux plans différents de l'imaginaire: le champ de la géométrie, une donnée qui se dissout progressivement, et le recours à une autre visée qui vient corriger - et sauver - la trajectoire du premier mouvement. Les dénominations de concentration et d'expansion que nous avions adoptées, permettent de rendre compte de ce double mouvement.

Reste une objection possible - qui en fait, n'en est pas une: comment expliquer que la partie du poème - trois vers - correspondant à l'expansion soit si courte, et vice versa? D'abord, ce n'est pas une contradiction, car c'est la strophe du champ imaginaire de la marche qui, par sa position stratégique, permet la relance du texte, suite à l'étouffement. Et si d'autre part, la concentration prend la part du lion, c'est qu'il s'agit là tout simplement d'une caractéristique de l'écriture - l'imagination garnienne ${ }^{6}$. A cet effet, il est assez révélateur de trouver dans l'édition critique des $\widetilde{C}$ uvres de Saint-Denys Garneau les ratures qui ont été opérées dans le corps de la première partie du poème: à titre d'exemple, 
je signale, à la strophe IV, la rature d'une double métaphore: l' "éclatement des bornes» trouvait une expansion métaphorique dans l'image d'un volcan, dont la lave devient métaphore de la vie intérieure?.

Le champ imaginaire de la géométrie renvoie bien à une figuration du cosmos, mais cette représentation, subordonnée au champ imaginaire, disparaît avant même la dissolution de cette visée formelle qui, de toute façon, est nettement prédominante.

Cette brève évaluation de la formalisation de l'imaginaire était nécessaire pour aborder le problème de l'énonciation, tant ces deux phénomènes - que nous dissocions pour des raisons méthodologiques - sont intimement liés dans le faire linguistique du texte.

Parler de l'énonciation, c'est s'interroger sur le mode d'inscription du sujet dans l'énoncé. Situons immédiatement notre hypothèse: le JE énonciateur se met en scène dans le poème pour s'y voiler et s'y dévoiler sous diverses figurations soumises à des opérations de transformation; c'est donc dire que ce texte, n'ayant pas de référent au sens classique du terme, constitue, non pas un simple miroir, mais un lieu problématique où s'inscrivent diverses traces du JE; ces inscriptions seront perçues comme relevant d'une procédure narcissique qui se caractérise par la multiplicité des figurations: le problème majeur qui nous préoccupera sera celui des interférences dans les renvois réciproques de ces images.

Dans cette perspective, le couple classique JE-énonciateur / JE-énoncé ne peut être retenu comme point de départ puisque cette corrélation apparaîtrait déjà comme une conclusion, une réponse évidemment trop générale à la question. II est méthodologiquement préférable d'aborder la question en relevant les figurations successives du JE.

En examinant le début du poème, on trouve au premier et au cinquième vers deux figurations: d'abord un JE biographique:

... j'ai fait des poèmes (v. 1)

puis un JE mythique:

... si j'étais le soleil... (v. 5)

Le premier renvoie à une figure actorielle et le second à une figure que, vu le long développement du champ imaginaire de la géométrie, nous nommons: actantielle.

Si l'on poursuit le fil du déroulement syntagmatique du poème, on trouve entre les vers 6 et 17 , une dominante du ON; du vers 18 au $25^{e}$, absence de sujet pronominal. Dans les trois vers suivants $(26,27,28)$ : 
l'apparition de celui qu'on nommera provisoirement: quelqu'un. Et du vers 29 à la fin, retour au JE, mais transformé en ME, c'est-à-dire une formule d'objectivation du JE.

Le JE mythique du vers 5 est le produit d'une métaphore bien marquée par la double marque rhétorique du «comme», et grammaticale du conditionnel:

... comme si j'étais...

Reprenons le terme d'expansion textuelle et imaginaire, apporté au JE biographique. Par la suite, la substitution du ON au JE et, dans une autre étape, la disparition du pronom sujet réalisent bien l'inverse de l'expansion, c'est-à-dire un mouvement de déconstruction ou, pour reprendre notre terminologie, de concentration. Suite à l'apparition du "quelqu'un", le sujet revient à la surface textuelle, mais objectivé; nous serions tenté de dire: «chosifié», mais pour ne pas déborder le seuil linguistique, nous dirons: substantivé.

Cette dernière manifestation du sujet est liée, sur le plan de l'imaginaire, au «réduit» (v. 33): il semble ici que ce plan de l'imaginaire vienne commenter le procès de l'énonciation en ce sens que le sujet réapparaît "réduit", mais n'anticipons pas en caractérisant dès maintenant le rapport entre les deux plans comme une relation herméneutique du commentaire: les rapports seraient à envisager comme pure fonction, c'est-àdire, un rapport bidirectionnel.

Une dernière constatation, dans la perspective formelle serait à faire: c'est qu'il se produit un décalage entre les deux plans analysés: les champs de l'imaginaire se développent dans la séquence d'une gouverne de la concentration, puis de l'expansion et enfin d'une synthèse des deux formes. Le plan de l'énonciation projette au début une expansion qui, se liquidant progressivement par la suite, réalise une forme de concentration jusqu'à la disparition du JE en tant que sujet d'un procès.

En termes linguistiques, on dira que le texte détruit la figure originaire du JE-acteur pour la textualiser, c'est-à-dire la transformer en figure actantielle qui, elle, est entraînée avec la liquidation du champ imaginaire de la géométrie.

Car c'est bien là le mouvement opéré sous les phénomènes de substitution et de transformation pronominales: le procès - perçu comme principe actif - se dissout pour aboutir, en fin de poème, à un statut. $\mathrm{Ce}$ qui nous permet maintenant de fonder notre proposition: dans le poème, Saint-Denys Garneau met en scène sa propre disparition, en tant que sujet énonciateur.

Notre hypothèse méthodologique consiste à considérer le texte comme le lieu d'un fantasme. Le fantasme, selon J.-B. Clément ${ }^{8}$, c'est la scène de la représentation, voire plus, la cause et la limite même de la représentation. Soulignons qu'il s'agit là de l'emprunt d'une perspective au 
champ de la psychanalyse et que ce transfert ne se fait pas sans adaptation: lorsque nous parlons de représentation, il ne s'agit pas d'un plan manifeste qui serait la simple projection - déformée, reformulée - d'un plan latent, mais bien d'un lieu unique que l'on analyse dans une perspective immanente; les opérations de transformation, loin d'être déduites, sont affichées dans le déroulement même du texte: voire plus, c'est justement ce travail - au sens psychanalytique du terme - qu'accomplit le texte dans sa dimension temporelle ou, plus proprement linéaire. Or, pour paraphraser J.-B. Clément, on dira que Saint-Denys Garneau manifeste sa propre exclusion.

L'origine et la limite de la représentation sont réalisées par les deux figures originaire et finale du JE d'abord valorisé par sa mythification l'image du soleil - puis dévalorisé du JE substantivé. Dans le poème, entre le JE originaire et le ME final, aucune inscription directe du JE: c'est d'abord ON, formule qui marque la "dépersonnalisation" et qui annonce l'absence de personne qui caractérise le passage suivant; il n'y a pas de formule intermédiaire pour le JE, ce qui nous ramène à la notion fondamentale en linguistique d'unités discrètes: l'embrayeur JE devient substantif et, en fin de compte, la référence ${ }^{9}$ unique. Il s'agit là d'un phénomène de "désembrayage" qui se réalise par une substantivation.

Dans une page du journal de Saint-Denys Garneau, on trouve ce passage fort suggestif:

Le verbe est un mot en devenir de substantif. Et l'on peut dire que le substantif est le verbe par excellence. C'est en lui qu'un passage trouve l'éternité de sa présence. Comme toute. forme changeante est à la recherche de son moment immobile, où l'agitation de son mouvement trouve le repos d'une parfaite solution en soi-même ${ }^{10}$.

On trouve exactement cette dissolution du procès en un statut. Et le poème se termine sur cette visée:

Pour vivre et l'art. (v. 34)

Cette formule, peu orthodoxe sur le plan grammatical, est d'autant plus révélatrice qu'en règle générale, les poèmes de Saint-Denys Garneau sont rigoureusement corrects, grammaticalement. Alors, s'agit-il d'une élision d'un «y», ce qui donnerait:

\section{Pour y vivre...}

formule qui rétablirait le sujet. Vu la contiguitté syntagmatique de «art", on peut, avec autant de vraisemblance,voir dans "vivre" un substantif au sens du partitif «vivre». Pourquoi alors, dira-t-on, l'auteur n'a-t-il pas écrit tout simplement: «la vie". Et justement l'infinitif "vivre" reste ambigu, introduisant et rejetant du même coup le sujet, donc le procès. C'est précisément ce phénomène auquel nous avons fait allusion plus haut en proposant le texte comme une scène où le sujet se voile / se dévoile. 
D'autre part, l'«art "renverrait au véritable sujet énoncé avec on ne peut plus de force, d'un nouveau procès qui est obnubilé: c'est le IL du pouvoir, la nécessité formelle du poème, le substitut, après transformation, du JE initial qu'on avait qualifié de biographique. Un IL pouvoir d'écriture-d'imagination qui fait avancer le texte, le "soleil", le IL. de la science la géométrie - le IL au pouvoir qu'il faudrait rétroactivement lire sous le "quelqu'un" de "Tel un homme..." qui, sur le plan de la rhétorique et de l'imaginaire permettait la relance du texte en le prenant en charge. Si bien que le JE biographique du début du poème, après le travail du fantasme, produisant une représentation, aboutit à un clivage du sujet, se scindant en un IL au pouvoir et un JE substantivé: ME.

Et nous touchons là un modèle de réalisation de l'énonciation assez courant dans la poésie de Saint-Denys Garneau que l'on peut présenter par le schéma phrastique suivant: un verbe intransitif central précédé du sujet JE et suivi d'un nouveau sujet, substitué au premier, qui réalise d'une façon implicite le IL. Ainsi, dans "Autrefois", le procès central réalise le devenir - ou la transformation - que l'on peut retracer à plusieurs endroits dans le poème; par exemple:

Comme si j'étais le... (v. 5)

Il me faut devenir... (v. 29)

Le procès trouve sa figuration la plus nette, et son aboutissement, dans le substantif "réduit». Au JE initial, correspond en terme de substitution, la formule neutre du «il... faut...", cette figure grammaticale de la nécessité étant reprise, en rejet, sous la forme syntaxique de la finalité:

Pour vivre et l'art.

Cette dernière transformation a comme effet d'obnubiler le procès causal et de reporter indéfiniment le sujet IL qui se cache sous la métaphore idéaliste du mot «art».

Un autre poème, "L'avenir nous met en retard", réalise, avec plus de netteté ce programme de nature syntaxique et sémantique:

L'avenir nous met en retard

Demain c'est comme hier on n'y peut pas toucher

On a la vie devant soi comme un boulet lourd

Le vent dans le dos nous écrase le front contre l'air aux talons

On se perd pas à pas

On perd ses pas un à un

On se perd dans ses pas

Ce qui s'appelle des pas perdus ${ }^{11}$.

Les oppositions sémantiques de l'espace (devant / derrière) et du temps (passé / avenir) suscitent et empêchent un devenir; cette contradiction se résout sur un autre plan: celui des figures pronominales où, encore ici, le NOUS (une variante du JE) cède la place aux pronoms neutres ON / 
SE et, en fin de texte, au CE, démonstratif neutre, antéposition.du syntagme figé: "pas perdus». Comme "l'art» de l'autre poème, le cliché langagier voile / dévoile le IL.

Nous pouvons maintenant reprendre notre renvoi initial au "Monologue fantaisiste sur le mot». Nous avions retenu une chaîne d'équivalence entre: MOT-MOI-MORT, puis enveloppé. Retenons qu'il s'agissait là d'une sorte de rêverie - le titre est bien: "fantaisie" - où Saint-Denys Garneau se fait, toutes proportions gardées, poéticien. Le Saint-Denys Garneau poète va plus loin en disposant autrement les termes: le poème nous révèle, par les deux bouts de la chaîne de transformation, une polarisation: MOT-MORT: le poème débute par le pôle MOT ( «... j'ai fait des poèmes") et se termine sur une MORT symbolique: le "réduit"; et c'est le JE (ou: MOI), en tant que trace textuelle, qui apparaît, dans le poème, comme véhicule, se déplaçant d'un pôle à l'autre.

Cette transformation du schéma, de l'essai au poème est rendue possible par l'intervention du travail textuel qui opère sur le plan de l'imaginaire: en fin de compte, c'est la dissolution du champ de la géométrie qui nécessite cette opération et, inversement, on dira que c'est la substantivation du sujet - transformation du procès en statut - qui sous-tend la transformation du champ de l'imaginaire.

Pour le MOI, il n'y a qu'une alternative: MOT-MORT; aucune situation intermédiaire n'est possible. Et l'ensemble des poèmes de SaintDenys Garneau met en scène de tels mouvements de transfert, d'un pôle à l'autre. Ainsi “Paysage en deux couleurs sur fond de ciel'12 " se termine par le vers suivant:

Un trou d'oubli - ciel calme autour

C'est la brèche, la béance, l'impossibilité d'une position intermédiaire, qui serait la seule confortable. Je lis au hasard ces autres vers:

Toutes paroles me deviennent intérieures ${ }^{13}$

C'est la fusion, cette fois, du MOT-MOI. Et ailleurs:

Pour mourir de soi-même tranquillement ${ }^{14}$

c'est l'autre point de fixation.

Saint-Denys Garneau n'est pas le poète idéaliste, voire mystique qu'on a dit; au contraire, l'écriture poétique en fait un dialecticien et ce, d'une façon rigoureuse, d'autant plus que cette dialectique domine tant le plan de l'imaginaire que la question de l'énonciation: dans les deux cas, le fonctionnement rhétorique est le même. Cette proposition ne surprendra 
pas si l'on se rappelle que Saint-Denys Garneau fut un ardent lecteur de Pascal, Racine et de leurs descendants spirituels que furent les romanciers chrétiens comme Mauriac, Bernanos et autres ${ }^{15}$... Nous faisons évidemment allusion à l'ouvrage de L. Goldmann sur la vision tragique du monde ${ }^{16}$.

Et je ne crois pas qu'on exagérerait l'affiliation en affirmant que la plupart des poèmes de Saint-Denys Garneau constituent un pari sur l'existence du MOI.

Jacques Blais ${ }^{17}$ proposait, pour parler du texte garnien, le principe d'une «distanciation ironique», cet espacement se lisant dans les jeux de substitution pronominale; nous sommes d'accord sur l'importance capitale de ce phénomène de distanciation qu'il fut d'ailleurs le premier à signaler. Mais, au sujet de l'ironie, le critique parle d'une cironie métaphysique", dont le poète aurait été la victime; encore ici, l'image du souffredouleur revient à la surface comme s'il était impossible de s'en débarrasser. Le concept de métaphysique appliqué à Saint-Denys Garneau est absolument faux; c'est à la dialectique que conduit la distanciation.

Pour revenir à nos propositions antérieures, nous dirons que la représentation de l'oscillation constante du MOl entre les deux pôles de la MORT et du MOT réalise une situation ambivalente de voilement / dévoilement qui se marque dans le déplacement continuel, le pôle MOT réalisant un dévoilement et à l'inverse le pôle MORT produisant l'effet de voilement. Ce déplacement constant qui, dans la dynamique du texte peut se lire dans les mouvements de concentration et d'expansion trouve ses formules les plus parfaites, parce que paradoxales, dans le «trou d'oubli » et dans le "réduit" qu'on peut maintenant relire: espace où la relation du JE au IL peut être à la fois d'assujettissement et de substitution, le procès étant «littéralement" obnubilé.

Les principales analyses thématiques de la poésie de Saint-Denys Garneau - et ici je pense aux travaux de G. Marcotte et de J. Blais ${ }^{18}$ ont toutes révélé un univers sémantique et thématique de la contradiction. Notre brève analyse pourrait nous conduire à considérer le $\mathrm{MOI}$ comme une figure thématique au même titre que, par exemple, l'os, la cage, l'envol de l'oiseau, etc., qui ne seraient alors que la projection symbolique du MOI en constante contradiction avec lui-même. Cette perspective "mimétique» - selon laquelle le texte, ici le poème, constitue un reflet, la communication d'un sens pré-déterminé - aurait l'avantage de rendre les analyses thématiques fonctionnelles au plus haut degré.

Mais inversons la perspective pour adopter l'optique sémiotique: le poème devient à l'égal du fantasme dans le rêve, le lieu de réalisation d'une production de sens: ici, un espace où la représentation du MOI, inscrite dans le JE et les autres symboles, trouve à la fois son origine et sa limite; plus que simple représentation, c'est le seul lieu où le JE se donne une consistance - une substance - qui ne peut d'ailleurs être que conflictuelle. Et comme on l'a suggéré plus haut, il ne s'agit pas d'une simple 
indécision, mais bien de l'écartèlement caractéristique d'une situation tragique.

Dans ces conditions, à quoi correspond le geste d'écriture, si ce n'est dans cet effort de voilement / dévoilement; Saint-Denys Garneau n'écrivait-il pas dans son journal qu'il faut arriver à " habiter le paysage ${ }^{19}$ ". "Habiter le paysage", c'est s'y enfouir, s'y cacher pour se retrouver, et ainsi infiniment...

Seulement le paysage, ce n'est pas celui touffu de nos forêts, mais celui des aquarelles, des mots (la "forêt des symboles" de Baudelaire). Ce paysage, lieu de délices, c'est la poésie, le poème enveloppe du MOI; d'où l'enjeu de ce texte: conférer une paternité au JE, trouver à situer le JE dans un espace créé artificiellement: le "réduit». La figure du pouvoir c'est l'«art", c'est le IL qui demeure implicite face au MOl. Là est le "mystère du poème" comme il le disait, ce va-et-vient dialectique qui ne peut s'inscrire que dans le poème, à l'exclusion de tous les autres types d'écriture.

Ce n'est qu'en tenant compte de ces considérations, me semble-t-il, qu'on peut vraisemblablement aborder le texte poétique garnien: comme un lien conflictuel entre l'essai où le JE énonciateur fonde son énoncé-objet et le journal intime où, à l'inverse, c'est le moi référentiel qui cherche à conférer une existence au sujet mythique, le JE énonciateur.

Et ce n'est pas un fait du hasard que Saint-Denys Garneau ait abordé ces trois types d'écriture: c'est à cette seule condition que sa poésie, telle que nous la connaissons, c'est-à-dire fondée sur la problématique du șujet, est possible: à la fois contradiction et synthèse des deux genres. Ne demandons plus pourquoi Saint-Denys Garneau n'a pas réussi à mener à bonne fin ses nombreux projets de romans ${ }^{20}$ : l'inscription du IL ne peut être qu'implicite chez lui alors que dans le récit, la figure du IL est prédominante.

En ce sens, le texte poétique tend vers l'impossible: inscrire l'inexistence du JE par le biais d'un triple conflit: JE / MOT, JE / MORT, JE / IL.

II peut être tout à fait révélateur de jeter un bref coup d'œil sur deux autres poésies pour y retrouver, par comparaison, les modes d'inscription du sujet.

Chez Nelligan par exemple, je lis dans "le Vaisseau d'or" un JE initial implicite qui est associé dans un rapport allégorique au vaisseau et qui se perd avec lui dans le naufrage. Mais si ce rapport JE-vaisseau demeure implicite jusqu'au rejet, un autre JE transcende le poème: celui enrichi jusqu'à l'extrême par les sèmes de la richesse, de l'or, du précieux, 
etc., mais aussi, en corrolaire, par les sèmes de la fragilité, de l'impossibilité de l'existence, réalisée par le naufrage. Nous discernons donc deux mouvements superposés de concentration (jusqu'au naufrage) et d'expansion (jusqu'à la figure mythique).

Cette double figuration du JE ne pouvait au début du siècle, que correspondre à l'image éthérée que l'on se faisait du «poète maudit». La transcendance de ce modèle ne pouvait d'ailleurs pas ne pas écraser la personnalité de l'adolescent qu'était Nelligan.

On a proposé, comme interprétation du célèbre sonnet, que le poète y préfigurait sa fin - ce qui ne veut absolument rien dire. Au contraire, c'est en inscrivant dans le poème son rêve de transfiguration pour coller à cette image mythique que Nelligan réalisait sa perte. Je vois dans ce cas, l'exemple le plus clair de ce phénomène que Michel Pêcheux nomme l'assujettissement de l'individu à une idéologie ${ }^{21}$ ou, ici, à une image transcendante, partie de l'idéologie.

Le poème apparaît donc comme lieu du fantasme où le JE-énonciateur se détruit, en tant que sujet autonome pour se transfigurer, sous un IL transcendant, implicite.

Chez Grandbois, et je retiens le poème «Fermons l'armoire... ", on trouve des séries de figures assez complexes sur le plan de la rhétorique que je nommerai de la nécessité, renvoyant autant à la prosodie du discours poétique qu'aux thèmes du prophète et de la magie; ce premier programme thématique est enchevêtré à un second où le JE sous la figure amoureuse, demeure immuable. L'expérience amoureuse est incessamment rompue et recommencée dans la poésie de Grandbois, mais il s'agit là d'une illusion de sens car la dimension temporelle est proprement absente; si l'on trouve des mécanismes de transformation, c'est qu'ils. ont comme fonction de nier le déroulement temporel; alors l'expérience amoureuse, perçue comme lieu d'inscription du sujet nous apparaît comme un déni des figures de la nécessité. Dans notre problématique, on dira que le JE se maintient dans sa figure initiale; voire plus, le travail du texte consiste justement à maintenir intacte cette image mythique du JE qui se profile victorieusement entre les IL(es de la nuit). Le texte se fonde donc sur deux mouvements parallèles d'expansion (la nécessité et le JE), de sorte qu'aucun conflit n'est possible.

En comparaison avec celles de Nelligan et de Grandbois, la poésie de Saint-Denys Garneau est celle qui inscrit avec le plus d'acuité la situation conflictuelle du JE. Si l'on prend comme acquis que chaque discours poétique constitue une solution originale au problème de l'énonciation, on verra chez Nelligan un mouvement d'expansion totale du JE énonciateur qui finalement se dissout et se perd dans les bascules métaphoriques successives. Chez Grandbois, deux mouvements parallèles d'expansion permettent la survivance d'un JE réalisant par là une sorte d'équilibre artificiel. Alors que chez Saint-Denys Garneau les figures de l'ex- 
pansion (métaphores) ne se manifestent qu'avec parcimonie, ne se prêtant que pour répondre à un minimum nécessaire au déroulement textuel; c'est que Saint-Denys Garneau ne cède en rien aux charmes - et aux facilités - de l'envol métaphorique qui, depuis les surréalistes est devenu la tarte à la crème de la poésie moderne.

II est d'ailleurs assez révélateur qu'en dehors de la poésie, Nelligan ne nous ait laissé aucun écrit et que Grandbois nous ait donné des nouvelles et un récit historique.

Cette écriture garnienne, en est-elle une de la castration, de l'impuissance comme on l'a souvent dit? Le renversement du sujet sur lui-même marque-t-il tout simplement une tendance narcissique rendue à un stade pathologique? Ou n'est-ce pas là la marque d'un effort de "conscientisation " dont l'exigence était proportionnelle à son effort de négation?

Nous avons proposé ici de projeter sur le texte poétique une problématique empruntée au champ de la psychanalyse; il semble que le recours au schéma du narcissisme, conjugué à la perspective linguistique où le déroulement discursif apparaît comme un faire soit, sinon nécessaire, du moins fort utile à l'élucidation de la question du sujet.

Si la poésie lyrique constitue un lieu exemplaire de l'inscription du sujet, ce que nous avons retenu comme point de départ, il n'en demeure pas moins des interrogations fondamentales: comment résoudre le problème des relations entre les JE intra- et extra-textuels? La sémiotique se donne comme programme de considérer le texte comme lieu de production de sens; alors posons la question naïve: à qui s'adresse ce sens? Et dans la poésie lyrique par surcroît? Ou plutôt: la perspective de la communication est-elle nécessaire? Évitable? Nous n'avons tenté ici qu'une réponse partielle: l'auteur considéré comme le premier lecteur. C'est d'ailleurs la perspective psychanalytique qui nous a entraîné sur cette voie. Mais cette solution, qui semble efficace dans le cas de SaintDenys Garneau, reste partielle. Et la question reste ouverte...

Au fait, nous n'avons peut-être réussi qu'à mieux poser le problème. 
1. Des pages du journal montrent Saint-Denys Garneau dans la crainte d'être "découvert" à la suite de la parution de Regards et jeux dans l'espace. II y a là le premier indice d'une volonté, chez le poète, de couper cette communication. Par contre, l'attention minutieuse que Saint-Denys Garneau portait à la présentation matérielle du recueil (publié à compte d'auteur) nous donne l'image d'un Saint-Denys Garneau lecteur de son propre texte. N.B. Toutes nos citations renvoient aux OEuvres, Montréal, PUM, 1970, «Bibliothèque des lettres québécoises". Quant à ce sujet de la "crainte d'être découvert ", voir le Journal, aux pages 495 et suivantes.

2. CEuvres, p. 26-27.

3. Ibid., p. 289-291.

4. Paris, Larousse, 1970 , "Langue et langage ", p. 117 et suivantes.

5. Ibid., p. 106 et suivantes.

6. Dans "Saint-Denys Garneau ou le procès métonymique " (Voix et images, vol. I, $n^{\circ} 3$, p. 432-441), Noël Audet démontre la dominante de cet axe dans la poésie de Saint-Denys Garneau.

7. «Dans le manuscrit, cette strophe (IV) se lit ainsi :

Alors la pauvre tâche

De pousser la périphérie jusqu'à la limite circonférence

Dans l'espoir que la surface du globe laisse ouvert

Un volcan par où déverser la lave

Brolante de toute cette bile, de ce rangement intérieur;

Dans l'espoir et d'un éclatement des bornes.

OEuvres, p. 1058.

Par quoi retrouver, libre, l'air et la lumière."n

8. "Le fantasme est à la limite de la représentation et de sa cause. [...] il est précisément la limite même." J.-B. Clément, "De la méconnaissance: fantasme, texte, scène” dans Langages, $n^{\circ} 31$, Paris, Didier-Larousse, septembre 1973, p. 37.

9. Et non le référent. Alors "référence" renvoie à un sens dépourvu de dénoté. II s'agit là d'un problème de logique soulevé par G. Frege: indiquons, à titre d'illustration, que le procès de vérité (vrai / faux) se pose au référent et non à la référence; ainsi, " roi de France", depuis la Révolution est devenu "référence». Voir, à ce sujet, J.- M. Adam, Linguistique et discours litterraire, Paris, Larousse, 1975, p. 12.

10. “Mot et culture» dans le Journal; OEuvres, p. 487.

11. Ibid., p. 183.

12. Ibid., p. $19-20$

13. Ibid., p. 156.

14. Ibid., p. 164.

15. Voir à ce sujet R. Bourneuf. Saint-Denvs Garneau et ses lectures européennes, Québec, PUL, 1969, "Vie des lettres québécoises", $n^{\circ} 6$.

16. Le Dieu caché, Paris, Gallimard, 1971, «Idées».

17. "Le Monologue ironique de Saint-Denys Garneau », dans De l'ordre et de l'aventure: la poésie au Québec de 1934 a 1944, Québec, PUL, 1975, "Vie des lettres québécoises", $n^{\circ} 14, p .141-164$.

18. G. Marcotte, le Temps des poètes, Montréal, HMH, 1969, p. 41-46; J. Blais, l'étude déjà citée et Saint-Denys Garneau et le mythe d'lcare, Sherbrooke, Cosmos, 1972.

19. «Propos sur l'habitation du paysage", dans les OEuvres, p. 674-676.

20. Dans une étude sur les récits-poèmes de Saint-Denys Garneau, Jean Gagnon expose ainsi la problématique du pronom-sujet IL: "Par voie de glissements et de permutation, il se voit remplacé par presque tous les autres pronoms de sorte qu'il s'annule finalement en tant que personnage puisqu'il a pris / emprunté tous les visages. II ne peut trouver son identité propre et / ou, s'il la trouvait, ce serait de n'en pas avoir puisqu'il est le «pauvre», le sans possession, le sans définition, le sans visage. De plus le $i l$ du pauvre se confond presque, dès le premier paragraphe, aves les il impersonnels et plus il se cherche, plus il s'indifférencie, aboutissant finalement à l'image la plus neutre, celle que nous portons tous en nous, qui nous porte tous: la acolonne vertébrale "." "ETC. ou Quand il est question d'une "prose" de Saint-Denys Garneau $"$, Voix et images, vol. $1, n^{\circ} 1, p .117$, note 7 .

21. Voir à ce sujet "Idéologie, interpellation. "effet Munchhausen", dans Michel Pêcheux, les Vérités de la Palice, Paris, Maspéro, 1975, p. 135-142. 